INVESTIGACIÓN

\title{
Errores frecuentes en el uso de la metodología de la investigación jurídica
}

\author{
Frequent errors in the use of the methodology of legal research
}

Rodrigo MARín VICHIS

Universidad Mexicana, México

\begin{abstract}
RESUMEN Sobre la base de la experiencia de veinte años en la cátedra del Derecho y cuatro como sinodal de exámenes orales, este trabajo reflexiona sobre los principales errores metodológicos en la presentación de diversas investigaciones jurídicas. Las confusiones son variadas y elegidas al azar con fines ilustrativos a modo de ejemplificar situaciones cotidianas en el mundo del aprendizaje y la educación jurídica contemporánea, y se proponen algunas de las posibles soluciones a fallos comunes, como lo es obviar la metodología de la investigación jurídica con la deducción, confundir la técnica procesal con la metodología de la investigación jurídica, considerarla como un accesorio para el estudio, citar de modo erróneo las fuentes, apropiarse indebidamente de creaciones ideológicas ajenas, igualar la información falsa, desestimar las fuentes indirectas de información, reducir la investigación al conocimiento al disponible en línea y utilizar de modo parcial los navegadores en la búsqueda exclusiva de la jurisprudencia o los reglamentos de las dependencias de gobierno. Precisar estos errores pueden propiciar la mejora de la calidad de los estudios.
\end{abstract}

PALABRAS CLAVE Metodología jurídica, investigación jurídica, enseñanza del Derecho.

ABSTRACT Based on the experience of twenty years in the chair of Law and four as a synodal of oral exams, this work reflects on the main methodological errors in the presentation of various legal investigations. The confusions are either varied and randomly chosen for illustrative purposes to exemplify everyday situations in the world of learning and contemporary legal education and propose some of the possible solutions to common failures are proposed, as it is to obviate the methodology of legal research with the deduction, to confuse it with the procedural technique, to consider it as an accessory, to worry little about the sources' accuracy, to misappropriate external ideological creations due to the incorrect use of the technology, to abound on internet sites with false information, disregard other indirect sources of information such as sites and anonymous comments, reduce the scope of knowledge to new online research and to partially use 
browsers in the exclusive search of jurisprudence or regulations of government agencies. Specifying these errors can lead to an improvement in the quality of the studies.

KEYWORDS Legal methodology, legal research, Teaching law.

\section{Antecedentes}

El contenido general de este artículo fue presentado el marco de primer Congreso Internacional de Metodología de la Investigación y Enseñanza del Derecho organizado por el Instituto de Investigaciones Jurídicas de la UNAM el 28 de noviembre de 2016. La experiencia surge de la actividad de veinte años como docente en la cátedra de Derecho ${ }^{1}$ en la Universidad del Valle de México y otras prestigiadas instituciones educativas, particularmente de los últimos cuatro años como sinodal en el Centro Nacional de Evaluación para la Educación Superior (Ceneval), asociación civil sin fines de lucro que desde 1994 ofrece servicios de evaluación a cientos de escuelas, universidades, empresas, autoridades educativas, organizaciones de profesionales de México y otras instancias particulares y gubernamentales. Su actividad principal es el diseño y la aplicación de instrumentos de evaluación. La Guía para el sustentante: Proceso para la acreditación de conocimientos a nivel licenciatura por acuerdo $286 y$ 328 (Ceneval, s/f) explica detalladamente las fases.

Para comprender la muestra de referencia es importante abundar brevemente sobre el funcionamiento de este centro. La Secretaría de Educación Pública ha facultado a este centro de evaluación para certificar conocimientos de estudios superiores, procedimiento que consiste en iniciar en una primera fase un Examen General para la Acreditación de Conocimientos Equivalentes a la Licenciatura (EGAL) en modalidad lápiz y papel. Luego, en la segunda fase, se invita al interesado a resolver un caso práctico relevante y vinculado a la licenciatura que se pretende acreditar y que se réplica de forma oral ante un grupo de sinodales. Ahí es donde he participado y he aprendido mucho por la colaboración de destacados colegas, como los profesores como Julio Hernández Pliego, José Barroso Figueroa, José de Jesús Ledesma Uribe, Angélica Carrera Dorantes, Maricela Moreno Cruz, Alejandro Torres y Enrique Cruz Villegas y Diana Lara Espinoza y Dulce Lozada, por mencionar a algunos.

En esta segunda fase, el sustentante justifica y argumenta de manera teórica-metodológica la solución que le dio al caso asignado y así demostrar los conocimientos y habilidades en su trabajo escrito de investigación. La resolución del caso práctico debe contener elementos fundamentales, como lo son la estructura y organización del texto, la ortografía, el marco teórico o de referencia que fundamenta la resolución

1. Esta experiencia me ha colocado en una situación de director, guía, facilitador y animador, como lo describe Martínez Miguelez (2014: 177). 
otorgada por el candidato, la metodología de la resolución, la calidad de respuesta, la información bien citada y fundamentada.

De aquí surge el fundamento y las muestras para precisar los principales errores metodológicos que he observado en la presentación de diversas investigaciones jurídicas.

\section{Introducción}

El problema de investigación que se trata de resolver en este estudio es que existen frecuentes errores metodológicos en la presentación de las investigaciones jurídicas de los estudiantes de Derecho derivados del contexto actual de interés en la materia, falta conocimiento tecnológico, insuficiente perspicacia y la apatía ética de los alumnos contemporáneos: obviar la metodología de la investigación jurídica con la deducción, considerarla como un accesorio para el estudio, equivocarse al citar las fuentes, cometer errores en el uso de la tecnología, igualar la información falsa, desestimar las fuentes indirectas de información, reducir el ámbito del conocimiento al disponible en línea, buscar de modo parcial los conocimientos o prescindir de los créditos pertenecientes a otras creaciones ideológicas. Este estudio procede de sucesos reales tangibles que se han tenido a la vista. Se proponen algunas soluciones para superar estas carencias que provienen de situaciones cotidianas en el mundo del aprendizaje y la educación jurídica actual. Se justifica porque señalar estos errores frecuentes pueden anticipar en el camino del estudio una mejor fluidez y ligereza, junto con ofrecer la ventaja de evitar tropiezos y ayudar a continuarlo sin mayores obstáculos.

Por eso, el objetivo general es describir la problemática de los errores metodológicos que presentan con frecuencia los alumnos estudiosos del Derecho al entregar sus investigaciones jurídicas. Los objetivos específicos son señalar y distinguir estos errores al resolver casos jurídicos y ofrecer algunas propuestas de solución.

La metodología que se utilizó para estas reflexiones fue el método cualitativo, en particular el estudio de trabajos entregados para ser evaluados, la mayor parte relativos a la resolución de casos prácticos y dilemas por resolver. Al finalizar, se ofrecen algunas conclusiones que reflexionan en temas comunes útiles para ser llevados a la práctica.

El error, en el sentido de esta investigación, se vincula a la parcialidad de un conocimiento y la creencia de que ha sido colmado el estudio, lo que suele generar una subjetiva y falsa expectativa de éxito. Se han tomado en consideración una centena de trabajos académicos durante el período de cuatro años, desde mayo 2013 a agosto de 2017, de alumnos principalmente de la Ciudad de México, pero también de otras regiones del país. El criterio con que se enumeran ha sido conforme a la cantidad en que fueron apareciendo. 


\section{Errores frecuentes en el uso de la metodología de la investigación jurídica}

\section{Obviar la metodología de investigación jurídica con la deducción}

En teoría, los argumentos de la verdad que se identifican con los deductivos derivan conclusiones ciertas de premisas del sentido común (Rojas Amandi, 2015: 15), mientras que la metodología de la investigación jurídica se fundamenta en elementos adicionales como la elección del tema, el proyecto de la investigación, el marco jurídico y conceptual, los métodos de búsqueda y análisis y las fuentes de la información (López Ruiz, 2014: 44).

Recibí en una ocasión un trabajo que no expresaba la metodología seguida. Cuando la investigación no especifica el método utilizado, frente a una pregunta expresa el sustentante señaló que había sido la deducción, tal y como hacen los juzgados. Parecía que con esa respuesta no había más que preguntar sobre la metodología, pues se había cerrado el panorama de investigación por completo. Pero al continuar con la exploración de sus conocimientos, y después de ser instruido y motivado para ampliar su perspectiva a fin de analizar el caso desde otros ángulos, comenzó a transfigurársele el rostro y su mente a maravillarse de cuántas cosas más podría haber descubierto.

En efecto, el método deductivo, ${ }^{2}$ utilizado por el sistema judicial en la resolución de las sentencias y quienes participan en él, considera como suficiente el hecho de realizar una exposición argumentativa lógica mediante las principales estructuras del silogismo para llegar a conclusiones inapelables. Sin embargo, este error limita a los estudiantes de Derecho y también a muchos juzgadores a considerar como suficiente la interpretación del conocimiento en base a la simple argumentación legal soportada en los diversos reglamentos y leyes, sin profundizar en las causas sociales, históricas, circunstancias psicológicas o carencias culturales que pudieran influir en el peso de los hechos o los motivos de las conductas, lo que sesga la aplicación normativa a un solo ángulo en el litigio.

Esta problemática abunda en la evaluación de muchos trabajos que han llegado a mis manos. Quienes pretenden acreditar las asignaturas correspondientes creen ilusamente que una buena investigación o desarrollo de un caso es justificado con un simple análisis procesal deductivo. Pero todos sabemos que la realidad jurídica es mucho más compleja: es necesario considerar las circunstancias de las partes, el contexto de saturación de las autoridades, la eficiencia de los acuerdos, el tiempo de las resoluciones, el soporte económico de nuestros clientes y la calidad de la defensa de los contrarios. Son contextos que sin duda hacen variar cualquier esquema deductivo enfocado exclusivamente en el procedimiento.

2. El método deductivo general se encuentra sintéticamente referenciado en López Ruiz (2014: 55). 
Por eso se propone, como la figura del búho que ilustra a nuestra profesión, mantenerse con los ojos bien abiertos sobre los asuntos de día y, de noche, desarrollar la actitud crítica (Baena, 2000: 17) para distinguir todas las variantes que pudieran influir en el resultado.

\section{Confundir la técnica procesal con la metodología de la investigación jurídica}

Doctrinalmente, la técnica procesal es diferente a la metodología de la investigación jurídica, pues la primera aplica sólo los ordenamientos legales de manera expresa, mientras que la segunda utiliza todos los conocimientos de las áreas del derecho público, privado y social ${ }^{3}$ para resolver un asunto.

Un sustentante, quien se desempeñaba como funcionario del poder judicial, ofreció su investigación con un detallado análisis técnico. No cabe duda de que el conocimiento sistemático era de muy alta calidad, pero la solución parecía no tener sentido, ya que perdía el objetivo de las partes. La solución más próxima y sencilla en los supuestos que se le ofrecieron a resolver era la conciliación, a fin de evitar a toda costa la violencia en cualquiera de sus formas.

De ahí que el proceso y el irrestricto apego a los supuestos generales - y me refiero a los títulos de los tópicos de los problemas legales establecidos en los reglamentos, como «despido injustificado», «divorcio» o «robo»- no son las únicas llaves para resolver los asuntos ni siempre los más adecuados. ¡Cuántas huelgas se han detenido! ¡Cuántos despidos se han evitado! ¡Cuántos actos de violencia se han impedido! Muchos y no siempre sobre el carril judicial, sino por arreglos por fuera.

Una prueba tangible de ello puede ser verificada en el mismo sistema de administración de justicia contemporáneo, que recientemente ha incorporado la justicia alternativa y la mediación para solución de conflictos.

Por esta razón, se propone abrir la veta de la doctrina, el análisis de los teóricos y el estudio de las corrientes ideológicas que influyen directamente en cada caso particular, para considerarlas durante todo el proceso.

\section{Considerar el requisito metodológico como un accesorio a la investigación y no como el eje soporte de la misma}

La investigación se define como «el conjunto de procesos sistemáticos y empíricos que se aplican al estudio de un fenómeno o un problema» y que aplicados de modo cuidadoso, sistemático y empírico genera conocimiento válido (Hernández Sampieri, Fernández y Baptista, 2014: 18).

Un trabajo contenía letras de una tipografía diferente cuando mencionaba la me-

3. Esta clasificación es más amplia en Suprema Corte de Justicia de la Nación (2006: 5). 
todología empleada. Era evidente que resultaba ser un añadido a última hora. Efectivamente, con frecuencia reviso trabajos que anexan como un añadido la mención metodológica utilizada de modo más o menos conceptual.

Cuando se solicita un procedimiento científico clásico que nos haga pensar que podría ser creíble la argumentación vertida en cada investigación, las personas simplemente expresan el nombre genérico de algún proceso metodológico que les resulte familiar y omiten detallar cuándo y dónde lo han aplicado. Esto trae como consecuencia que, en primer lugar, no siempre resulte ser el método expresado el mismo que se ha utilizado para la investigación y, en segundo lugar, se corre el peligro de evocarlos sólo al inicio para cubrir el requisito y omitir su aplicación. Por ello se propone observar bien ${ }^{4}$ y pormenorizar la aplicación del método en las diversas argumentaciones, a fin de que se vayan desarrollando. Por ejemplo, en este párrafo el método cualitativo, el estudio de casos, ha sido el modo en que he tratado de ordeñar el conocimiento expresado.

\section{Evocar fuentes y preocuparse poco por la exactitud}

La identificación de la fuente de la información es el punto de partida de los argumentos. Por ello es indispensable registrar el lugar preciso de donde proviene.

Después de leer un excelente trabajo, se pretendió ahondar en el tema, pero no se encontraron las fuentes de donde provenía la información. No cuesta nada identificar y tomar nota de los libros y fuentes que se usan en la investigación. Alumnos de Derecho con muy buena voluntad revisan sitios de internet que les generen ideas, pero olvidan tomar nota de esos lugares. Es algo relativamente sencillo cuando se utiliza la metodología apropiada, aunque también es un trabajo a veces tedioso y complicado. Se parece un poco al arte de cocinar, hay que vigilar la sal, la textura y la cantidad de fuego para que la sazón sea al gusto universal del paladar.

Hay quienes piensan que el evaluador nunca se va a tomar la molestia de verificar las citas. Es un ejercicio extra sin duda, pero que garantiza la calidad de las investigaciones.

\section{El riesgo de apropiarse indebidamente de creaciones} ideológicas ajenas a causa del uso incorrecto de la tecnología

La tecnología actual permite tener acceso a múltiples sitios de información. Sin embargo, esto no significa que quien realiza la investigación necesariamente la genere, ya que podría provenir de otras creaciones ajenas.

4. Miguel Martínez Migueles desarrolla un texto interesante sobre el método hermenéutico (Martínez Miguelez, 2014: 100). 
Una vez escuché a unos alumnos que decían «todo está en internet, cópialo, pégalo y cambia el formato». No hay nada como aparentar que uno sabe lo que ignora.

Los párrafos que ofrece la red son inconmensurables. Este tesoro tan abundante puede hacernos creer que no hay nada nuevo bajo el sol y que todo ya está escrito. En parte es cierto, todos nos alimentamos de las ideas de nuestros predecesores, pero, así como las huellas dactilares son distintas en cada ser humano, del mismo modo la capacidad de proceso de las ideas y de la información que nos brinda el mundo virtual puede ser significativamente útil y adecuado para cada mente.

Un error recurrido es el plagio de sitios de internet, el robo de la creación de otros y el apropiamiento de las ideas ajenas. Un buen cibernauta puede tener capacidades superiores al verificador de los trabajos y podría simular creaciones auténticas cuando no las hay.

Se dice que no hay mejor remedio para el mal que el bien, tampoco hay verdad que no salga a la luz, aunque tarde mucho. Aun cuando el evaluador puede ser engañado - lo que sabemos sucede con relativa frecuencia, pues se aceptan de buena fe muchos trabajos que podrían ser repeticiones de otros datos-, lo cierto es que nunca se podrá defraudar a la propia integridad.

El robo de la información adjudicada a sí mismo es una garantía para el límite personal que obstaculiza la capacidad para generar más. Cuando este secuestro de información se evidencia en los exámenes, entonces se presume que la investigación no es propia.

\section{Abundar en los sitios de internet con información falsa}

En teoría no es posible aceptar como válidas las conclusiones provenientes de argumentos falsos. Con frecuencia los alumnos no pueden distinguir las fuentes de información reales de las falsas.

Un alumno pretendía convencerme de una disposición legal respecto a la prueba paternidad y su relación al ADN. Desafortunadamente, la corte había hecho pronunciamientos encontrados y el sitio de consulta aseguraba resolver algún conflicto legal de quien ingresase a esa página, la cual, por cierto, tenía elementos que resguardaba el anonimato de su localización física. El conocimiento no sólo estaba equivocado, sino que, además, como solicitaba información confidencial, probablemente pudieran ser usados con fines ilícitos. A la hora de la presentación oral recomendé bloquear y denunciar el sitio.

Una tentación es acudir a las primeras páginas que coincidan con la búsqueda de la información de interés. Para evitar este escollo es importante también valorar los sitios que la ofrecen. Para ello, existen algunos parámetros clásicos que presuponen un filtro convencional, como son las páginas del gobierno, las universidades y centros educativos de prestigio y algunas empresas conocidas. 
Es evidente que, además del tamiz de datos, hay que tener cuidado con las páginas publicitarias, páginas "piratas» ${ }^{5}$ que simulan ser los sitios de instituciones financieras o de gobierno, lugares que incluso ofrecen información veraz pero muy reducida a cambio de ser direccionados a la publicidad que ellos mismos promueven. Hay que considerar los fenómenos sociales interrelacionados (Rojas Soriano, 2006: 51).

Hay un escollo cuando se restringe la aceptación a estas páginas y se descartan per $s e$ los blogs personales o generales: hay que reconocer que el conocimiento se genera en todas partes, pero también es necesario distinguir a muchos los defraudadores.

\section{Desestimar otras fuentes indirectas de información como los sitios y comentarios anónimos}

En teoría el conocimiento no tiene fronteras, pero con frecuencia los estudiantes no aprecian los contextos que circundan la problemática a resolver.

Un sustentante aseguró que no había más que investigar sobre el caso a desarrollar y que había agotado el tema en su totalidad. El sínodo comenzó a cuestionar el asunto desde el ámbito internacional y pusimos en evidencia cómo diversas legislaciones asumen ópticas distintas a una misma problemática, lo que con el tiempo evolucionó en el proceso legislativo también en nuestro país.

Hoy en día el anonimato es un medio de expresión revolucionario que ha permitido la evidencia de fraudes, delitos y de miles de situaciones irregulares. No obstante la necesidad de seleccionar las fuentes, no pueden menospreciarse trabajos, anécdotas y fuentes de información anónimas que por su contenido pueden diferenciarse de las falsas o fraudulentas. Por ejemplo, hay muchos comentarios en las redes que permiten comprender algunas corrientes ideológicas en contra de los partidos políticos, acciones de gobierno o hechos internacionales. Se ha reconocido a la denuncia anónima. Sin embargo, no siempre y no todas las veces el anonimato cubre la identidad de quienes exponen errores, delitos, información falsa o fraudes graves. Por ese motivo, la investigación jurídica seria no debe permitir el desdén de fuentes que necesariamente sean identificables, por supuesto que esto requiere una gran seriedad y una referencia expresa que garantice la calidad y la honorabilidad de la investigación y el reconocimiento y retractación oportuna cuando los datos verificados no sean consistentes con la verdad. ${ }^{6}$

5. López Ruiz (2014: 110) señala métodos para evitar el plagio.

6. Hernández Sampieri (2014: 207) nos ofrece una serie de recomendaciones para comprender la confiabilidad de los resultados y realiza análisis exhaustivos para las investigaciones cuantitativas mediante fórmulas, de donde se desprende la importancia de la seriedad de los resultados. 


\section{Reducir el ámbito del conocimiento a las nuevas tendencias de investigación en línea}

En teoría, los nuevos recursos tecnológicos amplían los espectros de investigación; pero también es claro que las mercadotecnias de los sitios promueven para su propio beneficio el acceso más simple, lo que no siempre coincide con los recursos más valiosos. En la investigación, si se desea obtener resultados más objetivos, es indispensable acercarse también a las bibliotecas, buscar sentencias, hemerotecas, acudir a seminarios y realizar observaciones entre otras técnicas para obtener información.

La solución no se encuentra en línea, me aseguraba un colega. Así fue: la investigación relativa a un presunto robo no tenía ninguna huella en la red respecto de los actos violentos de un lugar.

Hay quienes piensan que la vida se encuentra dentro del teléfono inteligente o de la tableta. Por supuesto que hay más datos de información fuera de la realidad virtual. Aun cuando todavía no maduramos la avalancha de información que inunda nuestras pantallas y terminales, no menos cierto es que la vida fuera de las cajas electrónicas ofrece el rastro de datos con frecuencia más impactante y verosímil a primera vista. ${ }^{7}$

Se utilizan de modo parcial los navegadores en la búsqueda exclusiva de la jurisprudencia o los reglamentos de las dependencias de gobierno

De manera académica no se puede desdeñar un trabajo sustentado en tesis jurisprudenciales o reglamentos oficiales, ya que ofrecen sin duda un valor jurídico particular. Sin embargo, estas referencias podrían sesgar los estudios y reducirlos a consideraciones muy parciales, propias de resoluciones administrativas o judiciales.

Un sustentante centró la solución a su caso exclusivamente en el proceso jurídico vigente, pero no consideró que las víctimas de un delito tuvieron el acierto de verter al escrutinio público el modus operandi de quienes se aprovecharon de su buena fe, lo que podría dar resultados más eficientes para restituir su derecho.

En efecto, como se dijo antes, la denuncia anónima ciudadana en las dependencias de gobierno y ONG son ya una institución. Pero las costumbres juristas orillan a los investigadores a destacar como lugares preferidos de consulta la Cámara de los Diputados o la Suprema Corte de Justicia, así como las páginas de la Secretaría de Gobernación, Hacienda o los tribunales de justicia.

7. También existen diversas técnicas para el procesamiento de datos (Hernández Sampieri, 2014: 439). 


\section{Conclusiones}

Un investigador jurídico serio amplía su espectro a cualquier información relevante sobre su asunto. La reseña de una película, un hecho noticioso, la aparición de un libro, las declaraciones de una víctima, las conversaciones de personas relacionadas a una situación de interés son elementos que no pueden dejar de ser considerados ni omitidos en la búsqueda del conocimiento.

El marco conceptual con que hemos partido distingue al método científico y algunos particulares errores en los que se puede caer en la investigación jurídica. La metodología que se ha utilizado muestra con claridad que muchos de estos desaciertos pueden ser subsanados y que podrían mejorar la calidad de los estudios legales. El análisis realizado sobre el riel de la metodología cualitativa respecto al estudio de casos ofrece un parámetro ilustrativo adecuado y significativo. Se han señalado frecuentes equívocos en el marco teórico considerados como errores, algunas causas comunes y los diversos riesgos a los que se encuentra expuesto un investigador de buena fe, principalmente una persona que se inicia en el ámbito de la educación jurídica. Estas precisiones pueden mejorar de la calidad de los estudios. Aun cuando este registro está limitado y no exhaustivo tanto por motivos de confidencialidad de las políticas de cada centro educativo y la protección de los datos personales de los casos en estudio, ilustra de manera fehaciente algunos vicios comunes y frecuentes en los que se puede enredar todo estudioso del derecho.

La metodología es el camino y no un fin, pero no se puede llegar a la meta sin recorrer una ruta.

\section{Referencias}

Baena Paz, Guillermina (2000). El análisis: Técnicas para enseñar a pensar y a investigar. Ciudad de México: Editores Mexicanos Unidos.

Ceneval (s/f). Guía para el sustentante: Proceso para la acreditación de conocimientos a nivel licenciatura por acuerdo 286 y 328. Ciudad de México: Ceneval. Disponible en http://bit.ly/2nywtlY.

Hernández Sampieri, Roberto, Carlos Fernández y Lucio Baptista (2014). Metodología de la investigación. 6. ${ }^{\mathrm{a}}$ ed. Ciudad de México: McGraw-Hill.

López Ruiz, Miguel (2014). Nuevos elementos para la investigación: Métodos, técnicas y redacción. $3^{\text {a }}$ ed. Ciudad de México.

Martínez Miguelez, Miguel (2014). Ciencia y arte en la metodología cuantitativa. Ciudad de México: Trillas.

Rojas Amandi, Víctor Manuel (2015). Argumentación jurídica. Ciudad de México: Oxford University Press México. 
RojAs Soriano, Raúl (2006). Guía para realizar investigaciones sociales. 13. ${ }^{\text {a }}$ ed. Ciudad de México: Plaza y Valdés.

Suprema Corte de Justicia de la Nación (2006). El sistema jurídico mexicano. Ciudad de México.

\section{Sobre el autor}

Rodrigo Marín Vichis es licenciado en Derecho y maestro en Derecho Corporativo por la Universidad Mexicana, maestro en Ciencias de la Familia por el Instituto Superior de Estudios para la Familia, doctorando en Derecho Penal y postgraduado en Derecho por el Centro de Estudios Superiores en Ciencias Jurídicas y Criminalísticas.Su correo electrónico es marinvilchis@gmail.com. 


\title{
REVISTA PEDAGOGÍA UNIVERSITARIA Y DIDÁCTICA DEL DERECHO
}

La Revista Pedagogía Universitaria y Didáctica del Derecho (RPUDD) es una publicación científica semestral que contribuye tanto a la reflexión multidisciplinaria sobre Pedagogía Universitaria y Didáctica del Derecho, para la formación y consolidación de esta área de investigación, como a la difusión de prácticas innovadoras en la enseñanza-aprendizaje del Derecho, considerando el contexto nacional e internacional..

\author{
DIRECTORA \\ María Francisca Elgueta Rosas \\ ASISTENTE EDITORIAL \\ Priscilla Saavedra Medina \\ SITIO WEB \\ pedagogiaderecho.uchile.cl \\ E-MAIL \\ rpedagogia@derecho.uchile.cl \\ LICENCIA DE ESTE ARTÍ́CULO
}

Creative Commons Atribución Compartir Igual 4.0 Internacional

\author{
9 \\ La edición de textos, el diseño editorial \\ y la conversión a formatos electrónicos de este artículo \\ han estado a cargo de Tipográfica \\ (www.tipografica.cl).
}

